\title{
Autonomous Operators for Direct Use on Irregular Image Data
}

\author{
S.A. Coleman ${ }^{1}$ and B.W. Scotney ${ }^{2}$ \\ ${ }^{1}$ School of Computing and Intelligent Systems, University of Ulster, Northland Road, \\ Londonderry, BT48 7JL, Northern Ireland \\ ${ }^{2}$ School of Computing and Information Engineering, University of Ulster, Cromore Road, \\ Coleraine, BT52 1SA, Northern Ireland \\ \{sa.coleman, bw.scotney\}@ulster.ac.uk
}

\begin{abstract}
Standard image processing algorithms for digital images require the availability of complete, and regularly sampled, image data. This means that irregular image data must undergo reconstruction to yield regular images to which the algorithms are then applied. The more successful image reconstruction techniques tend to be expensive to implement. Other simpler techniques, such as image interpolation, whilst cheaper, are usually not adequate to support subsequent reliable image processing. This paper presents a family of autonomous image processing operators constructed using the finite element framework that enable direct processing of irregular image data without the need for image reconstruction. The successful use of reduced data (as little as $10 \%$ of the original image) affords rapid, accurate, reliable, and computationally inexpensive image processing techniques.
\end{abstract}

\section{Introduction}

In image processing it is common to consider images as regular lattices of twodimensional samples. However, irregularly sampled images can arise from motion or disparity compensation, such as in motion-compensated video coding, motion compensated video interpolation or disparity-compensated interpolation in stereoscopic images [9]. Irregularly spaced image data also occur frequently in areas such as remote sensing [11], medical imaging, oceanography and human retinal perception, [2, 3]. For example, in human retinal perception, human photoreceptors are not regularly distributed but have a personal signature denoted by the random positioning of cells. Hence, in order to replicate human retinal perception, use of irregular image data needs to be supported. Also, in underwater acoustic imaging systems, where an image is obtained by transmitting acoustic waves and sensing the waves reflected by objects using an array of sensors, various conditions may arise that lead to the array sensor data being irregular.

The application of standard image processing algorithms to irregularly sampled images often results in unreliable results. The underlying difficulty is that many such algorithms require the availability of complete, and regularly sampled, image data. This means that such algorithms are often applied to complete images that have been 
reconstructed from sparse irregularly sampled, and noisy data without a priori knowledge of the image content. Image reconstruction techniques include methods based on: cubic spline representation of the image [9]; wavelet representation of the image [10]; the theory of projections onto convex sets [8]. Other simpler techniques, such as low order image interpolation, whilst cheaper, are usually not adequate to support subsequent reliable image processing. Therefore a means of direct use of irregularly sampled images needs to be considered. Such an approach has a number of advantages including potential for efficient image coding [4].

In this paper we propose a family of autonomous image processing operators that can be applied directly to irregularly sampled images without the additional requirement of image reconstruction. These autonomous operators are able to change shape and size as required across the image plane through the use of adaptive Gaussian basis functions within the finite element framework. This approach has benefits with regard to reduced computational intensity and increased speed compared with methods that require full image reconstruction. In Sections 2 and 3 we introduce the finite element framework within which the autonomous operators are constructed. Section 4 presents the finite element implementation, which we call the Primrose algorithm, highlighting how the local operator size and shape are directly linked to the local neighbourhood point density. The efficient implementation of the operator design procedure is also discussed. In Section 5 we present the results of our algorithm using a little as $10 \%$ of the original image data.

\section{Irregular Data Representation}

We consider an irregularly sampled image to be represented by a spatially irregular sample of values of a continuous function $u(x, y)$ of image intensity on a domain $\Omega$. Our operator design procedure is then based on the use of a mesh generated using Delaunay triangulation [7].

With each node $i$ in the mesh is associated a piecewise linear basis function $\phi_{i}(x, y)$ which has the properties

$$
\phi_{i}\left(x_{j}, y_{j}\right)= \begin{cases}1 & \text { if } i=j \\ 0 & \text { if } i \neq j\end{cases}
$$

where $\left(x_{j}, y_{j}\right)$ are the co-ordinates of the nodal point $j$ in the mesh. Thus $\phi_{i}(x, y)$ is a "tent-shaped" function with support restricted to a small neighbourhood centred on node $i$ consisting of only those triangles that have node $i$ as a vertex; $\phi_{i}$ is linear on each mesh triangle. We then approximately represent the image function $u$ by a function $U(x, y)=\sum_{j=1}^{N} U_{j} \phi_{j}(x, y)$ in which the parameters $\left\{U_{1}, \ldots, U_{N}\right\}$ are mapped from the image intensity values at the $N$ irregularly located nodal, or scatter, points. The approximate image function representation is therefore piecewise linear on each triangle and has value $U_{j}$ at node $j$. 


\section{Weak Form of Autonomous Operators}

We formulate autonomous image operators that correspond to weak forms of operators in the finite element method $[1,5,6]$. Operators used for smoothing may be based simply on a weak form of the image function, for which it is assumed that the image function $u(x, y)$ belongs to the Hilbert space $H^{0}(\Omega)$; that is, the integral $\int_{\Omega} u^{2} d \Omega$ is finite. Feature detection and enhancement operators are often based on first or second derivative approximations, for which it is necessary that the image function $u(x, y)$ is constrained to belong to the Hilbert space $H^{1}(\Omega)$; i.e. the integral $\int_{\Omega}\left(|\underline{\nabla} u|^{2}+u^{2}\right) d \Omega$ is finite, where $\underline{\nabla} u$ is the vector $(\partial u / \partial x, \partial u / \partial y)^{T}$.

Corresponding to a first directional derivative $\partial u / \partial b \equiv \underline{b} \cdot \underline{\nabla} u$ or a second directional derivative $-\underline{\nabla} \cdot(\mathbf{B} \underline{\nabla} u)$, we may use a test function $v \in H^{1}(\Omega)$ to define the weak forms

$$
E(u)=\int_{\Omega} \underline{b} \cdot \underline{\nabla} u v d \Omega \quad \text { and } \quad Z(u)=-\int_{\Omega} \underline{\nabla} \cdot(\mathbf{B} \underline{\nabla} u) v d \Omega .
$$

Here $\mathbf{B}=\underline{b} \underline{b}^{\mathrm{T}}$ and $\underline{b}=(\cos \theta, \sin \theta)$ is the unit direction vector. Zero-crossing methods are often based on the isotropic form of the second order derivative, namely the Laplacian $-\underline{\nabla} \cdot(\underline{\nabla} u)$. This is equivalent to the general form in which the matrix $\mathbf{B}$ is the identity matrix $\mathbf{I}$.

In the finite element method a finite-dimensional subspace $S^{h} \subset H^{1}$ is used for function approximation; in our design procedure the irregular image $U$ is a function in $S^{h}$, and $S^{h}$ is defined by the irregular mesh of triangular elements and piecewise linear basis functions described in Section 2.

Since we are focusing on the development of autonomous operators that can explicitly embrace the concept of size and shape, our design procedure uses a finitedimensional test space $T_{\sigma}^{h} \subset H^{1}$ that explicitly embodies a size parameter $\sigma$ that is determined by the local scatter point density. This generalisation allows sets of test functions $\psi_{i}^{\sigma}(x, y), i=1, \ldots, N$, to be used when defining autonomous derivative based operators; for first and second order operators respectively, this provides the functionals

$$
E_{i}^{\sigma}(U)=\int_{\Omega} \underline{b}_{i} \cdot \underline{\nabla} U \psi_{i}^{\sigma} d \Omega \text { and } Z_{i}^{\sigma}(U)=\int_{\Omega} \underline{\nabla} U \cdot\left(\mathrm{B}_{i} \underline{\nabla} \psi_{i}^{\sigma}\right) d \Omega
$$

\section{Design Procedure}

The test space $T_{\sigma}^{h}$ comprises a set of Gaussian basis functions $\psi_{i}^{\sigma}(x, y), i=1, \ldots, N$ of the form 


$$
\psi_{i}^{\sigma}(x, y)=\frac{1}{2 \pi \sigma^{2}} e^{-\left(\frac{\left(x-x_{i}\right)^{2}+\left(y-y_{i}\right)^{2}}{2 \sigma^{2}}\right)} .
$$

Each test function $\psi_{i}^{\sigma}(x, y)$ is restricted to have support over a neighbourhood $\Omega_{i}^{\sigma}$, centred on node $i$, consisting of those triangular elements that have node $i$ as a vertex. We note therefore that the integrals in the definitions of the functionals $E_{i}^{\sigma}$ and $Z_{i}^{\sigma}$ can be computed by integration over only the neighbourhood $\Omega_{i}^{\sigma}$ rather than the entire image domain $\Omega$, providing the functionals

$$
E_{i}^{\sigma}(U)=\int_{\Omega_{i}^{\sigma}} \underline{b}_{i} \cdot \underline{\nabla} U \psi_{i}^{\sigma} d \Omega_{i} \text { and } Z_{i}^{\sigma}(U)=\int_{\Omega_{i}^{\sigma}} \underline{\nabla} U \cdot\left(\mathrm{B}_{i} \underline{\nabla} \psi_{i}^{\sigma}\right) d \Omega_{i} \cdot
$$

\subsection{Autonomous Local Size Selection}

The usually difficult issue of local size selection for an operator is now naturally addressed by the distribution of the nodes in the mesh-based representation of the image. For a scatter point $\left(x_{i}, y_{i}\right)$ we obtain the local operator size directly from the mesh in the neighbourhood $\Omega_{i}^{\sigma}$. We consider an approach which we have named the Primrose algorithm.

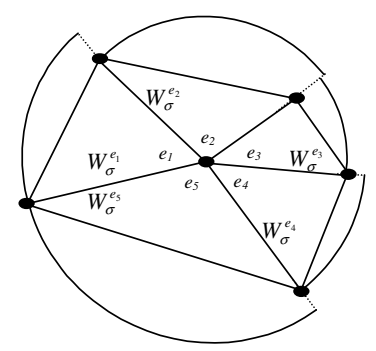

Primrose Operator

Fig. 1. Neighbourhoods $\Omega_{i}^{\sigma}$ in irregular mesh

In the Primrose algorithm the neighbourhood $\Omega_{i}^{\sigma}$ is defined to have a real-valued "radius" $W_{\sigma}^{e_{m}}$ for each element $e_{m}$ in $\Omega_{i}^{\sigma}$. In each case $W_{\sigma}^{e_{m}}$ is chosen as the radius of the smallest circle centred on $\left(x_{i}, y_{i}\right)$ containing element $e_{m}$. Each element therefore contributes a "petal" to the Primrose operator as illustrated in Figure 1. The test function $\psi_{i}^{\sigma}$ is correspondingly comprised of a set of sectors of Gaussian functions $\psi_{i}^{\sigma_{m}}$, where $\psi_{i}^{\sigma_{m}}$ is the test function over element $e_{m}$ in $\Omega_{i}^{\sigma}$. In each case choosing the element scale parameter $\sigma_{m}=W_{\sigma}^{e_{m}} / 1.96$ ensures that along the longest element edge of $e_{m}$ through $\left(x_{i}, y_{i}\right) 95 \%$ of the cross-section of the Gaussian is contained in $e_{m}$. 
Construction of these autonomous operators on an irregular grid differs significantly from construction of image processing operators on a regular grid in that it is no longer appropriate to build explicitly an entire operator as in [6]; each operator throughout an irregular mesh is autonomous and may be different with respect to the operator neighbourhood size, shape, and the number of nodal points in the operator. When using an irregular grid, we work on an element-by-element basis to build each operator, taking advantage of the flexibility offered by the finite element method as a means of adaptively changing the irregular operator size and shape to encompass the data available in any local neighbourhood. Such local neighbourhoods are illustrated by the collections of triangular elements shown in Figure 2; in each neighbourhood the test function $\psi_{i}^{\sigma}$ is comprised of a set of sectors of Gaussian functions $\psi_{i}^{\sigma_{m}}$ truncated at "radius" $W_{\sigma}^{e_{m}}$. Thus each operator is able to automatically alter its shape and size as required, dependent on the irregular node placement corresponding to the sampling of the image data. Operator $a$ in Figure 2 has a central node with 5 adjoining nodes, operator $b$ illustrates 7 adjoining nodes and operator $c$ illustrates 6 adjoining nodes.

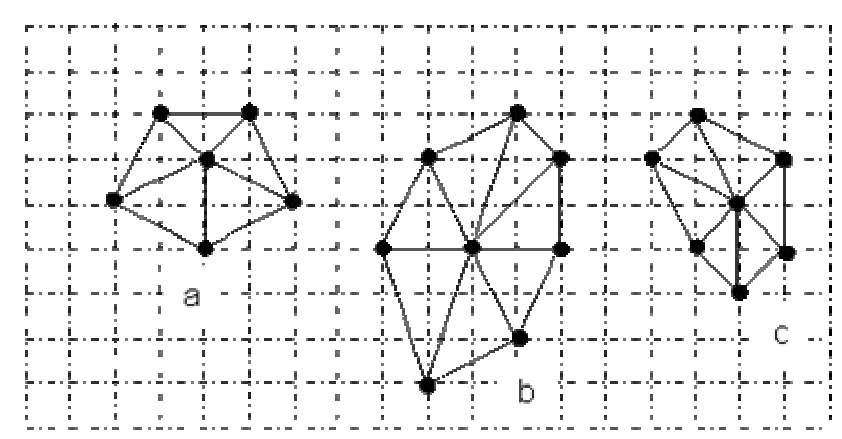

Fig. 2. Neighbourhoods of Autonomous Operators

\subsection{Efficient Implementation}

The spatial relationship between the node $i$ on which the Gaussian basis function $\psi_{i}^{\sigma}$ is centred and each of the nodes in the neighbourhood $\Omega_{i}^{\sigma}$ is readily available from nodal numbering and locational information routinely stored in the finite element method. The integrals required in the computation of operators such as $E_{i}^{\sigma}(U)$ and $Z_{i}^{\sigma}(U)$ are evaluated through the standard process of finite element assembly: integrals $k_{i s}^{m, \sigma}$ over each element $e_{m} \subset \Omega_{i}^{\sigma}$ are approximately evaluated using Gaussian quadrature rules, requiring function evaluations in $e_{m}$ of the test function $\psi_{i}^{\sigma}$ and of the three piecewise linear basis functions $\phi_{s}$ (locally indexed $s=$ 1,2,3) whose support includes element $e_{m}$. Since the first derivatives of the piecewise linear basis functions are locally constant, each element integral may be accurately approximated by just four function evaluations (i.e., using a four-point Gauss rule). 
The issue of evaluating integrals over irregularly shaped triangular elements is routinely handled in the finite element method by the use of isoparametric mappings that relate each element to a "standard" right-angled triangle on which numerical integration can be efficiently and accurately performed [1].

\section{Results}

To obtain results for our proposed technique, we simulated irregular image data by randomly selecting a specified proportion of an underlying $256 \times 256$ pixel intensity image. Such a sample is in no way based on a prior knowledge of the original image content. In order to apply our proposed technique to irregular image data, we initially use Delaunay triangulation to generate a triangular mesh in which the irregular image data points are nodes. As the nodal point set simply corresponds to the co-ordinates of the irregular image data, the local density of nodes in this mesh is simply controlled by the local availability of data points. This mesh is then used as the basis for the application of the finite element based autonomous operators described in Sections 3 and 4 . Figure 3 illustrates an original $256 \times 256$ image and the corresponding randomly generated irregular image data and Delaunay triangulation.

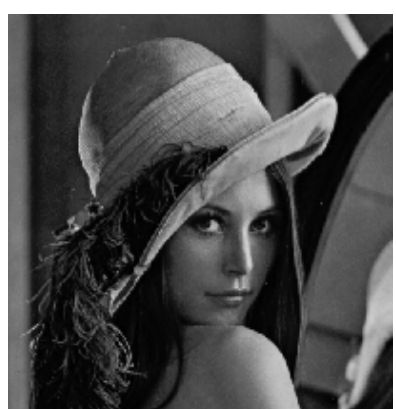

(a)

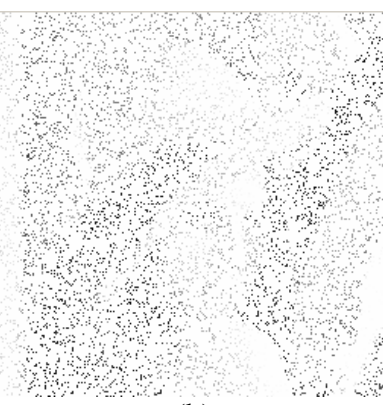

(b)

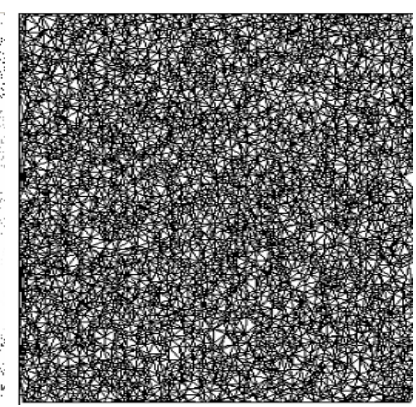

(c)

Fig. 3. (a) Original image (b) Irregular image data using a $10 \%$ sample (c) Delaunay triangulation

The autonomous operators are applied directly using the mesh illustrated in Figure 3(c) in order to generate a feature point set.

Figure 4(a) illustrates the thresholded feature point set generated when our family of autonomous operators corresponding to the first derivative operators $\left\{E_{i}^{\sigma}\right\}$ are applied to the triangular mesh illustrated in Figure 3(c). Already we can see the outline of the features appearing in this feature point set. To generate a more complete feature map, a simple edge linking technique was applied based on the similarity of edge direction between neighbouring feature points, and the results of this are illustrated in Figure 4(b). Although the feature map is not perfect, enough information is available to distinguish the main features in the irregular image data without the additional expense of image reconstruction to generate a regular data set. 


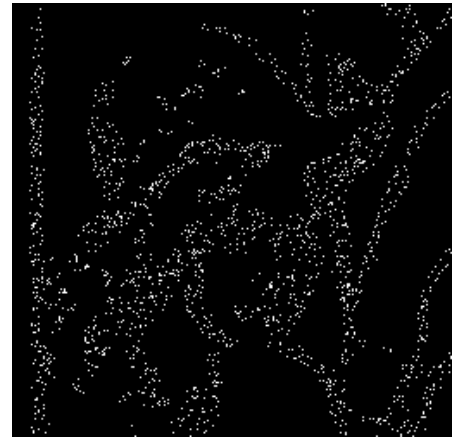

(a)

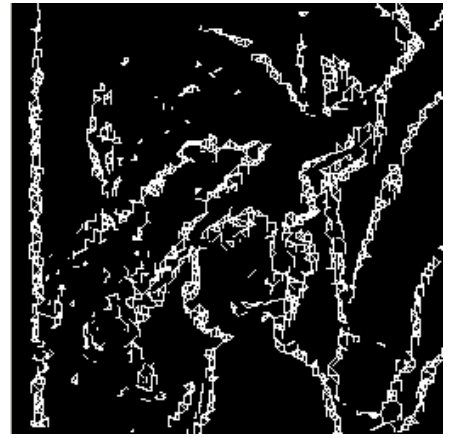

(b)

Fig. 4. (a) Feature point set generated from Figure 3(c); (b) Completed feature map

For further illustration, Figure 5 shows the same technique applied to $10 \%$ of another real image; again we can see that the main features appear quite clearly.

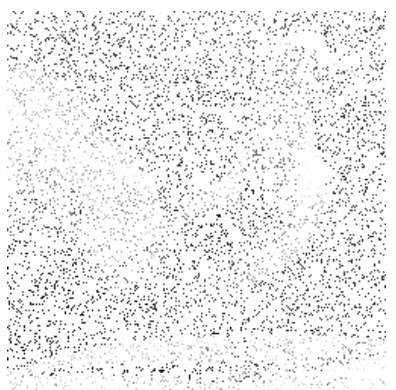

(a)

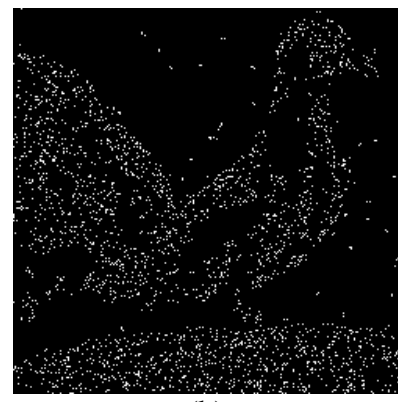

(b)

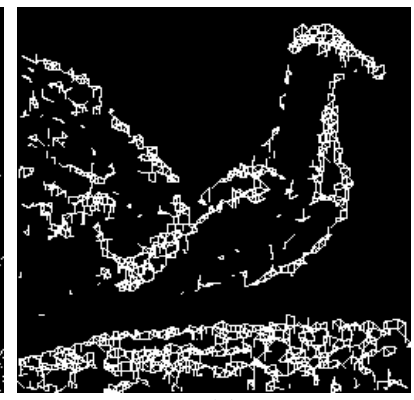

(c)

Fig. 5. (a) Irregular image data using a 10\% sample; (b) Feature point set; (c) Completed feature map

\section{Summary}

In applications that rely on the use of incomplete image data, the processing of images to extract features is problematic without the availability of supplementary information on image content. This is because standard feature detection algorithms usually require the availability of complete and regularly sampled image data. Techniques that are based on image reconstruction without prior image knowledge do not generally provide reliable mechanisms for accurate feature extraction. In this paper we have presented a family of autonomous image processing operators based on a generalisation of the finite element method that can naturally formulate design procedures that can be used to successfully implement feature detection directly on non-uniformly sampled images. 
Although this research currently uses a rudimentary edge linking technique, promising results have been presented, and further work will entail the application of the current operator design procedures to real images supported by more advanced edge linking algorithms. This problem will be explored for the dual purposes of feature extraction and for the further decomposition of the image into a very sparse content-based point set from which a reconstructed image may be cheaply yet accurately derived.

\section{Acknowledgement}

This work was funded by the Nuffield Foundation under the research grant number NAL/00839/G.

\section{References}

[1] Becker, E.B., Carey, G.F., and Oden, J.T., Finite Elements: An Introduction, Prentice Hall, London, 1981.

[2] Petrou, M., Piroddi, R., Chandra, S., "Irregularly Sampled Scenes" Proceedings of SPIE Image and Signal Processing for Remote Sensing, Vol., SPIE5573, 2004.

[3] Piroddi, R., Petrou, M., "Dealing with Irregular Samples" Advances in Imaging and Electron Physics, Vol.132, Elsevier, pp109-165, 2004.

[4] Ramponi, G., Carrato, S., "An Adaptive Irregular Sampling Algorithm and its Application to Image Coding" Image and Vision Computing, Vol.19 pp. 451-460, 2001.

[5] Scotney, B.W., Coleman, S.A., Herron, M.G., "A Systematic Design Procedure for Scalable Near-Circular Gaussian Operators.” Proc. IEEE ICIP, pp 844-847, 2001.

[6] Scotney, B.W., Coleman, S.A., Herron, M.G., " Device Space Design for Efficient ScaleSpace Edge Detection” Proc. ICCS, Amsterdam, LNCS 2329, Springer, pp1077-1086, 2002.

[7] Shewchuk, J.R., "Triangle: Engineering a 2D Quality Mesh Generator and Delaunay Triangulator" ${ }^{\text {st }}$ Workshop on Applied Computational Geometry, pp. 124-133, 1996

[8] Stasinski, R., Konrad, J., "POCS-Based Image Reconstruction from Irregularly-Spaced Samples" Proceedings of IEEE International Conference on Image Processing, pp. 315318, 2000.

[9] Vazquz, C., Dubois, E., Konrad, J., "Reconstruction of Irregularly-Sampled Images by Regularization in Spline Spaces" Proceedings of IEEE International Conference on Image Processing, pp. 405-408, 2002.

[10] Vazquz, C., Konrad, J.,Dubois, E., "Wavelet-Based Reconstruction of IrregularlySampled Images : Application to Stereo Imaging” Proceedings of IEEE International Conference on Image Processing, pp. 319-322, 2000.

[11] Yegnanarayana B, Mariadassou CP \& Saini P. "Signal Reconstruction from Partial Data for Sensor Array Imaging applications”, Signal Processing, Vol. 19, pp.139-149, 1990. 